\title{
Savannah River Site 1991 Road Erosion Inventory
}




\section{Table of Contents}

\begin{tabular}{|l|l|}
\hline & Pages \\
\hline Report with Maps & $1-6$ \\
\hline Table 1. Erosion Type and Cover Characteristics & 2 \\
\hline Table 2. Treatment by Erosion Type & 3 \\
\hline Table 3. Example of Top Two Rows of a Recording Form & 3 \\
\hline Table 4. Inventory Results by Type & 4 \\
\hline Map 1. 1991 SRS Road Erosion Inventory Results & 5 \\
\hline Map 2. 1991 SRS and Topography & 6 \\
\hline Documentation for Inventory Sheets on pages A-1 to A-19 & i \\
\hline Data for 1991 SRS Soil Erosion and Sediment Results & A-1 to A-19 \\
\hline
\end{tabular}




\section{Savannah River Site 1991 Road Erosion Inventory}

This paper explains the rationale and results of a 1991 road erosion inventory conducted by members of the USDA Forest Service - Savannah River (FS-SR) and USDA Natural Resources Conservation Service (NRCS). The inventory provided information for the Department of Energy - Savannah River (DOE) to justify the need for developing an erosion and sediment control program with appropriate funding, personnel, and equipment. Federally managed since the early 1950's, the SRS is located on 198,344 acres $(80,301$ hectares) in the South Carolina counties of Aiken, Barnwell, and Allendale. Located along the eastern border of the Savannah River, the SRS is located within the Upper and Lower Coastal Plains of South Carolina.

During normal operations U.S. Forest Service personnel observed soil erosion and sedimentation problems in connection with roads and facilities. Poorly vegetated road ditches, or other areas of land disturbances are among the most common erosion and sedimentation concerns. With increasing steepness and length of slope, erosion and sediment rates substantially increase. Large impervious areas of asphalt, concrete, rooftops, and compacted soils often associated with facilities and parking lots generate tremendous runoff velocities and volumes. While the accelerated runoff effects are not necessarily apparent around the facilities, a visual inspection of the receiving channels and bordering steep slopes might reveal runoff impacts like gullies and other erosion concerns.

Before and during the FS-SR program to stabilize borrow pits and other disturbed areas on a smaller scale, management realized the need to increase the program. Beginning in the late 1980's, NRCS participated on erosion control projects and DOE funded a fulltime Resource Conservationist employee in 1990 on the SRS. Personnel also observed sediment blowing across roads, which provided a safety concern.

Upon the 1991 arrival of the first staff officer for the newly created Soil, Water, \& Air (SWA) section, the FS-SR program increased, and had professional and technical assistance from the NRCS. The two units planned to conduct an erosion assessment with a desired result of increasing the soil stabilization program. Divided into road erosion and facility erosion, the assessment started in May 1991 and ended in August 1991. This paper only describes the erosion assessment related to roads or other isolated areas within view from the road. A facility erosion assessment conducted the same year is not discussed. The inventory results were presented to DOE for program development beginning with fiscal year 1992.

Primary, secondary, and forest roads (also called native surface roads) are the three common SRS road types. Small sections of roads are linked to various monitoring wells, or other industrial use. Many, if not most SRS employees, travel solely on paved primary roads to and from their homes to their industrial workplace. There are fairly straight secondary roads several miles long, which allow for fairly rapid transition to certain parts of the interior. While many secondary roads were found bare of vegetation during the inventory, many have since been graveled. With a cover of native soils, native surface 
roads are of various lengths. Those roads allow access to a specific stand or a set of stands used for various resource activities like forestry, wildlife, or research.

Though the total length of roads remained unknown at the beginning of the inventory, an estimate of 2,900 kilometers was given by management. A 2003 review of a 2000 roads electronic coverage in the geographic information systems reveals about 2,200 kilometers of roads on the SRS, or about $76 \%$ of the earlier estimate.

\section{Methodology}

Besides soil erosion, hardpan and sediment observations were noted along with a remarks section for general observations.

Two persons riding in government vehicles inventoried all roads, whether paved, gravel, or native surfaced. The persons noted the distance and width of road erosion. For borrow pits or other broad area the observers made on-site estimates, which they verified later with available aerial photography, if available. Field measurement methods varied. Longer distances of 500 feet or more were determined by vehicle odometer readings, while the observers estimated the shorter distances. Observers calibrated their visual ability to estimate distances by weekly comparisons of a length and width field estimate followed by a tape measure comparison.

Based on soils coverage characteristics (see Table 1), the survey identified four erosion types. On two observations, surveyors noted water ponding on the road from a flooded wetland, which were not identifiable under any erosion type. Noted erosion types assisted the development of treatments needs (see Table 2). Vegetative cover improvement would require the least intensive treatment, followed in intensity treatment levels by sheet erosion, rill erosion, and gully erosion.

Table 1. Erosion Type and Cover Characteristics

\begin{tabular}{|l|l|l|}
\hline Type Number & Erosion Type & Cover Characteristics \\
\hline 1 & Gully & $\begin{array}{l}\text { Bare soils; slope failure; scouring of } \\
\text { soils exceeding twelve inches in } \\
\text { width or depth. }\end{array}$ \\
\hline 2 & Rill & $\begin{array}{l}\text { Similar characteristics to a gully, } \\
\text { but six to twelve inches in width or } \\
\text { depth. }\end{array}$ \\
\hline 3 & Sheet & $\begin{array}{l}\text { Bare or very poorly vegetated soils. } \\
\text { Vegetative Cover }\end{array}$ \\
\hline Improvement & $\begin{array}{l}\text { Sparse to moderately vegetated } \\
\text { soils. }\end{array}$ \\
\hline
\end{tabular}

With a form, personnel immediately recorded observations (see Table 3). Normally estimated in feet, the length and width estimated were later calculated into acres in the office. Like mentioned previously, borrow pits or other observed areas of concern were initially field estimated before aerial photographs calculations in the office. 
Table 2. Treatment by Erosion Type

\begin{tabular}{|l|c|c|c|l|l|}
\hline & \multicolumn{5}{|c|}{ Recommended Treatments } \\
\hline Erosion Type & Lime & Fertilizer & Seed & \multicolumn{1}{|c|}{ Land Shaping } & \multicolumn{1}{c|}{ Cover Type } \\
\hline Gully & Yes & Yes & Yes & $\begin{array}{l}\text { Bulldozer, or other } \\
\text { medium to large } \\
\text { equipment. }\end{array}$ & $\begin{array}{l}\text { Heavy duty } \\
\text { erosion control } \\
\text { blankets; geo- } \\
\text { textile; or } \\
\text { structures. }\end{array}$ \\
\hline Rill & Yes & Yes & Yes & $\begin{array}{l}\text { Light shaping with } \\
\text { box blade or other } \\
\text { manual equipment }\end{array}$ & $\begin{array}{l}\text { Mulch; erosion } \\
\text { control } \\
\text { blankets. }\end{array}$ \\
\hline Sheet & Yes & Yes & Yes & Not normally & $\begin{array}{l}\text { Mulching } \\
\text { common. }\end{array}$ \\
\hline $\begin{array}{l}\text { Vegetative Cover } \\
\text { Improvement }\end{array}$ & Yes & Yes & Varies & None & $\begin{array}{l}\text { Possible spot } \\
\text { mulching. }\end{array}$ \\
\hline
\end{tabular}

Individual sheets were used for each timber compartment. Under the Site column, personnel identified the road number and marked the unit on natural resource compartment maps.

Table 3. Example of Top Two Rows of a Recording Form

Compartment Number: $\underline{40}$

\begin{tabular}{|l|l|l|l|l|l|l|l|}
\hline Site & Type & Distance & Width & Hardpan & Sediment & Remarks & Acres \\
\hline Road 9 & 4 & 400 & 30 & N & N & Only on east side of road. & 0.28 \\
\hline $\begin{array}{l}\text { Meyers } \\
\text { Mill } \\
\text { Road }\end{array}$ & 2 & 500 & 60 & N & Y & $\begin{array}{l}\text { Sediment moving toward } \\
\text { stream drainage. }\end{array}$ & 0.69 \\
\hline
\end{tabular}

Along with observations noted since 1991, the inventory data was recorded electronically in a watershed improvement needs database that no longer receives computer support. However, a hardcopy remains in the USFS-SR hydrologist's office. In 1994, a GIS layer created by on-screen digitizing using the U.S. Geologic Survey roads coverage for a reference is stored in the author's geographic information workspace. Despite the poor spatial accuracy of the coverage, it is useful and relates well to the roads coverage.

\section{Observations and Conclusions}

Throughout the survey, major erosion problems were often observed on native surface roads and rights-of-ways that transverse steep slopes. Frequently mowing also caused erosion concerns. North of Road A (Highway 125) the inventory persons observed more erosion sites and concerns because of the steeper and longer slopes combined with a greater amount of facilities and rights-of-ways.

The conclusion identified in Table 4, Map 1, and Map 2 requires knowledge of the details behind the observations. Individual observations often had more than one type of erosion concern. For example, the person may observe a gully mixed in with rills, or other observations. In Table 4, the list is based on the greatest concern identified at that observation. Gullies are the greatest concern, followed in order by sheet, rill, and 
vegetative cover improvement. For example, a one hectare area of sheet erosion and rills might contain a gully. In hectares, the gully area might total only 0.1 hectare with the rest of the site being a combination of other erosion types. Since gully erosion has the most intensive impact and greatest repair costs, the list places all the hectares under the gully category.

Table 4. Inventory Results by Type

\begin{tabular}{|l|c|c|c|c|c|c|c|}
\hline \multicolumn{2}{|c|}{} & Total & Average & Maximum & Minimum & $\begin{array}{c}\text { Standard } \\
\text { Deviation }\end{array}$ & Variance \\
\hline Type & Observations & $\mathbf{( H a )}$ & $\mathbf{( H a )}$ & $\mathbf{( H a )}$ & $\mathbf{( H a )}$ & $\mathbf{( H a )}$ & $\mathbf{( H a )}$ \\
\hline n/a & 2 & 0.10 & 0.05 & 0.08 & 0.02 & 0.05 & 0.00 \\
\hline Gully & 130 & 130 & 16.19 & 16.19 & 0.00 & 2.10 & 10.93 \\
\hline Rill & 110 & 48 & 8.50 & 8.50 & 0.00 & 0.97 & 2.31 \\
\hline Sheet & 106 & 75 & 7.28 & 7.28 & 0.00 & 1.22 & 3.72 \\
\hline VCI $^{1}$ & 123 & 49 & 3.44 & 3.44 & 0.00 & 0.53 & 0.69 \\
\hline Total & 471 & 302 & 16.19 & 16.19 & 0.00 & 1.38 & 4.69 \\
\hline
\end{tabular}

${ }^{1}$ Vegetative Cover Improvement

In brief, the inventory played an important role in producing SRS awareness to the need for establishing an erosion and sediment control program involving a budget; a broad range of labor, technical, contracting, and management skills; and cooperation among the interested contractors and federal agencies. All major roads and rights-of-ways were limed, fertilized, and seeded along with most of the grasses surrounding the facilities. Over the years, contract workers completed most of the erosion and sediment control projects. Forest Service employees, including summer students, also completed many projects.

By Cliff Jones, Hydrologist of the USDA Forest Service-Savannah River. June 22, 2007 


\section{SRS Road Erosion Inventory Results}

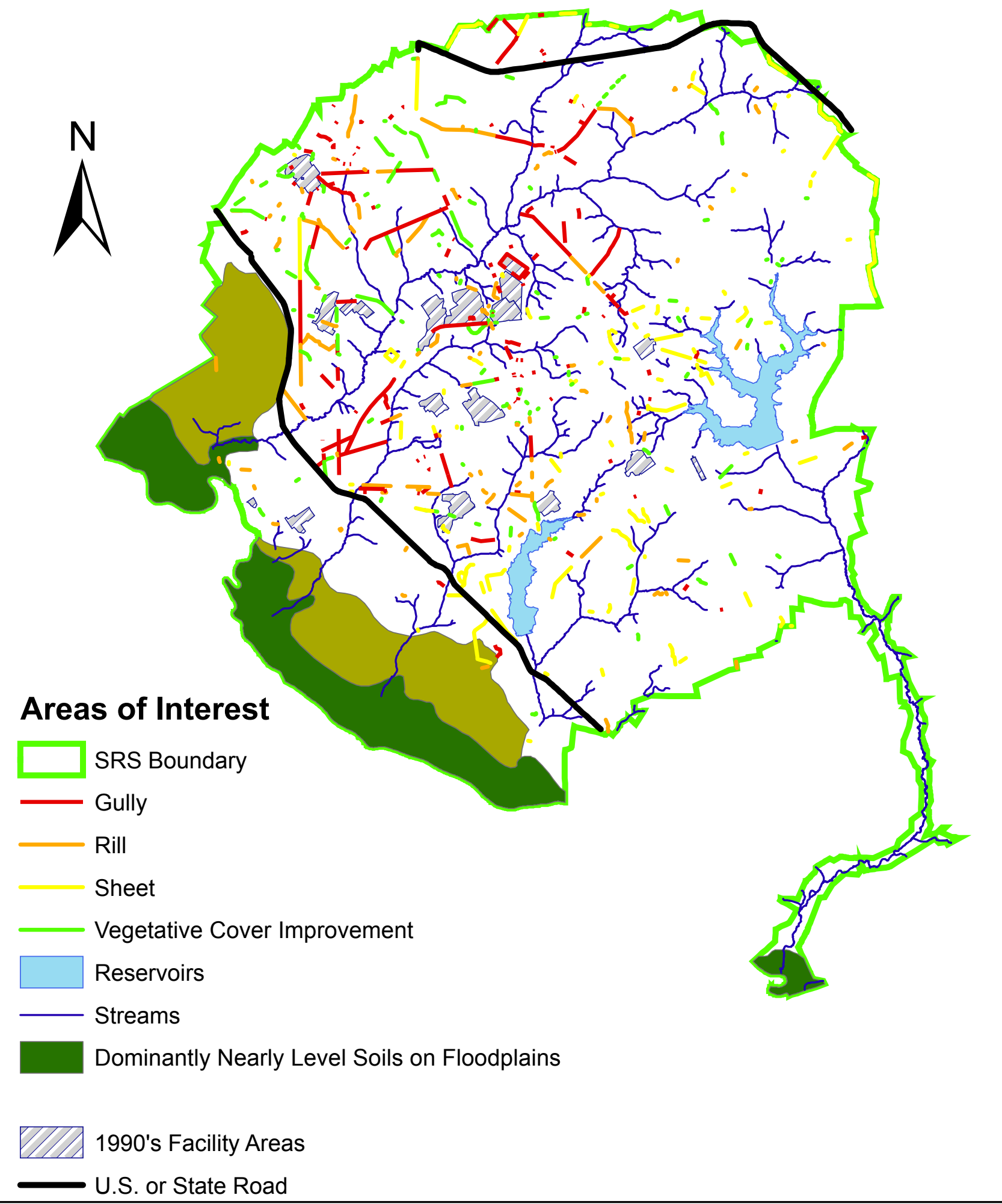




\section{SRS and Topography}

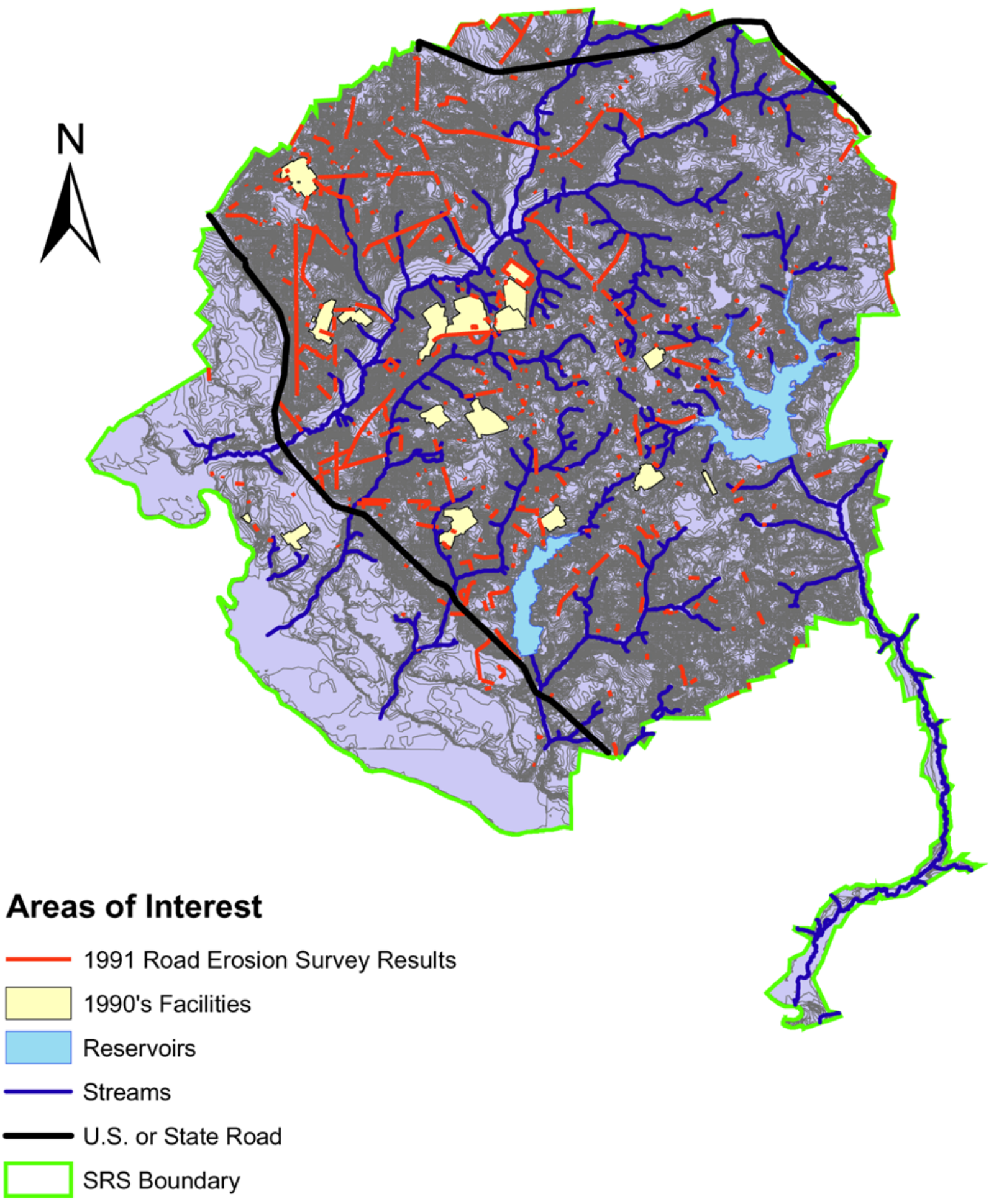


Pages A-1 to A-19 are the raw data for the 1991 SRS Road Erosion and Sediment Inventory

This section has the following.

GIS Code relates to a Geographic Information Systems identifier. Road Name lists the road or the nearest road. Comp. refers to the natural resources compartment.

Type, length, and width are previously identified in the document. Hardpan relates to soils hardened or cemented by iron oxide, silica, calcium carbonate, or similar substances.

Hardpan greatly inhibits the movement of precipitated water through soils. In the case of the SRS, the soils survey indicates that many of the soils have some siliceous content, including Dothan and Fuquay soils. These same two soils have some plinthite.

In the remarks section, ditch work is commonly listed. This remark relates to soil erosion in the roadside drainage ditches. The sand/soil removal remark listed in GIS Code 96 is a term used because we did not have exact knowledge of the reason for the removal of the sand or whatever other soils were removed. The area definitely relates to a large amount of soil removal, possibly a future landfill or a related industrial use. Sediment refers to soils transported by water runoff, wind, or gravity. Piles of soil that appeared to have been moved by equipment are noted.

Acres are calculated with the length and width data. 


\begin{tabular}{|c|c|c|c|c|c|c|c|c|c|}
\hline $\begin{array}{l}\text { GIS } \\
\text { Code }\end{array}$ & Road Name & Comp. & Type & \begin{tabular}{c|} 
Length \\
(ft)
\end{tabular} & $\begin{array}{c}\text { Width } \\
\text { (ft) }\end{array}$ & HP & Sed. & Remarks & Acres \\
\hline 1 & Off 5-26 & 5 & 1 & 50 & 10 & 0 & 1 & Ditch work & 0.0 \\
\hline 2 & $7-22$ & 7 & 1 & 500 & 30 & 0 & 1 & Ditch work & 0.3 \\
\hline 3 & $7-30$ & 7 & 1 & 2,640 & 30 & 0 & 1 & & 1.8 \\
\hline 4 & $5-20$ & 5 & 4 & 2,640 & 40 & 0 & 1 & Ditch work & 2.4 \\
\hline 5 & $D-1$ & 5 & 4 & 1,584 & 30 & 0 & 1 & Ditch work & 1.1 \\
\hline 6 & $D-1$ & 7 & 1 & 2,112 & 30 & 0 & 1 & Ditch work & 1.5 \\
\hline 7 & $5-29$ & 5 & 3 & 300 & 150 & 0 & 0 & & 1.0 \\
\hline 8 & $D-1$ & 7 & 2 & 500 & 60 & 0 & 0 & Ditch work & 0.7 \\
\hline 9 & $1-A$ & 7 & 4 & 1,056 & 30 & 0 & 0 & & 0.7 \\
\hline 10 & D-1 & 7 & 1 & 375 & 30 & 0 & 0 & Ditch work & 0.0 \\
\hline 11 & $9-25$ & 9 & 1 & 30 & 20 & 0 & 0 & & 0.0 \\
\hline 12 & 1 & 5 & 4 & 1,000 & 30 & 0 & 1 & & 0.7 \\
\hline 13 & $7-26$ & 7 & 2 & 300 & 30 & 0 & 0 & Ditch work & 0.2 \\
\hline 14 & $7-21,7-26$ & 7 & 1 & 6,336 & 30 & 0 & 1 & Ditch work & 4.4 \\
\hline 15 & $1-A$ & 9 & 4 & 1,056 & 30 & 0 & 0 & & 0.7 \\
\hline 16 & $5-33$ & 5 & 2 & 500 & 30 & 0 & 1 & Ditch work & 0.3 \\
\hline 17 & $7-26.1$ & 7 & 1 & 2,112 & 30 & 0 & 1 & Ditch work & 1.5 \\
\hline 18 & 1 & 5 & 4 & 1,056 & 30 & 0 & 1 & & 0.7 \\
\hline 19 & $9-28$ & 9 & 1 & 3,168 & 30 & 0 & 0 & Ditch work & 2.2 \\
\hline 20 & Near 7-33 & 7 & 4 & 40 & 30 & 0 & 1 & $\begin{array}{l}\text { Ditch work, ruts } \\
\text { in Rd }\end{array}$ & 0.0 \\
\hline 21 & $9-28$ & 9 & 1 & 1,200 & 30 & 0 & 1 & Ditch work & 0.8 \\
\hline 22 & C-1.1 & 4 & 2 & 3,168 & 40 & 0 & 0 & & 2.9 \\
\hline 23 & $9-28$ & 9 & 2 & 250 & 30 & 0 & 1 & Ditch work & 0.2 \\
\hline 24 & Off $4-29.3$ & 4 & 1 & 5,808 & 40 & 0 & 1 & & 5.3 \\
\hline 25 & 4-27 & 4 & 2 & 2,376 & 40 & 0 & 1 & $\begin{array}{l}\text { Steep slope on } \\
\text { one side }\end{array}$ & 2.2 \\
\hline
\end{tabular}




\begin{tabular}{|c|c|c|c|c|c|c|c|c|c|}
\hline $\begin{array}{l}\text { GIS } \\
\text { Code }\end{array}$ & Road Name & Comp. & Type & \begin{tabular}{|c|} 
Length \\
(ft)
\end{tabular} & $\begin{array}{c}\text { Width } \\
\text { (ft) }\end{array}$ & HP & Sed. & Remarks & Acres \\
\hline 26 & Off 4-27 & 4 & 4 & 500 & 50 & 0 & 1 & Logging area & 0.6 \\
\hline 27 & SC 125 & 10 & 4 & 1,100 & 50 & 0 & 0 & & 1.3 \\
\hline 28 & Near 3-24 & 3 & 4 & 800 & 10 & 0 & 0 & & 0.2 \\
\hline 29 & $\mathrm{Rd} C$ & 10 & 4 & 2,000 & 40 & 0 & 1 & & 1.8 \\
\hline 30 & C-1.1 & 4 & 3 & 100 & 50 & 0 & 1 & Deep-cut banks & 0.1 \\
\hline 31 & $4-26$ & 4 & 4 & 5,808 & 40 & 0 & 1 & & 5.3 \\
\hline 32 & C-1.1 & 10 & 2 & 400 & 30 & 0 & 1 & Ditch work & 0.3 \\
\hline 33 & Into $A-1.1$ & 2 & 2 & 225 & 20 & 0 & 0 & $\begin{array}{l}\text { Needs ditchwork; } \\
\text { discing }\end{array}$ & 0.1 \\
\hline 34 & Into A-4.7a3 & 6 & 2 & 0 & 0 & 0 & 1 & $1 / 2$ acre & 0.5 \\
\hline 35 & West of $6-33.1 \mathrm{a}$ & 6 & 3 & 100 & 50 & 0 & 0 & & 0.1 \\
\hline 36 & North 6-33.2 & 6 & 2 & 400 & 30 & 0 & 0 & & 0.3 \\
\hline 37 & $6-33$ & 6 & 4 & 500 & 30 & 0 & 0 & & 0.3 \\
\hline 38 & 3 & 6 & 2 & 100 & 30 & 0 & 0 & & 0.1 \\
\hline 39 & $6-42$ & 6 & 4 & 40 & 10 & 0 & 0 & & 0.0 \\
\hline 40 & A-4.7 & 6 & 4 & 300 & 225 & 0 & 1 & & 1.6 \\
\hline 41 & 2 & 19 & 2 & 750 & 30 & 0 & 1 & Ditch work & 0.5 \\
\hline 42 & 2 & 19 & 3 & 2,000 & 30 & 0 & 1 & Ditch work & 1.4 \\
\hline 43 & $8-23$ & 8 & 2 & 1 & 30 & 0 & 0 & $\begin{array}{l}\text { Wetland } \\
\text { encroaching }\end{array}$ & 0.0 \\
\hline 44 & $8-23$ & 8 & 4 & 500 & 30 & 0 & 1 & $\begin{array}{l}\text { Ruts in Rd; } \\
\text { drainage }\end{array}$ & 0.3 \\
\hline 45 & Above 8-29 & 8 & 1 & 250 & 40 & 0 & 1 & Ditch work & 0.2 \\
\hline 46 & Near 8-30 & 8 & 1 & 1,056 & 40 & 0 & 1 & & 1.0 \\
\hline 47 & $7-20$ & 7 & 2 & 5,808 & 40 & 0 & 1 & Ditch work & 5.3 \\
\hline 48 & $8-30$ & 8 & 1 & 200 & 40 & 0 & 1 & $\begin{array}{l}\text { Deep gullies; } \\
\text { steep slopes }\end{array}$ & 0.2 \\
\hline 49 & West of $8-30$ & 8 & 4 & 180 & 40 & 0 & 1 & Ditch work & 0.2 \\
\hline 50 & East of 8-30 & 8 & 1 & 600 & 10 & 0 & 1 & Steep wall & 0.1 \\
\hline
\end{tabular}




\begin{tabular}{|c|c|c|c|c|c|c|c|c|c|}
\hline $\begin{array}{l}\text { GIS } \\
\text { Code }\end{array}$ & Road Name & Comp. & Type & $\begin{array}{l}\text { Length } \\
\text { (ft) }\end{array}$ & $\begin{array}{l}\text { Width } \\
\text { (ft) }\end{array}$ & HP & Sed. & Remarks & Acres \\
\hline 51 & South of $8-22$ & 8 & 1 & 1,100 & 40 & 0 & 1 & $\begin{array}{l}\text { Deep gullies; } \\
\text { ditch work }\end{array}$ & 1.0 \\
\hline 52 & D-1 & 8 & 2 & 50 & 30 & 0 & 1 & Ditch work & 0.0 \\
\hline 53 & D-1 & 8 & 1 & 500 & 40 & 0 & 1 & High wall & 0.5 \\
\hline 54 & $8-21$ & 8 & 2 & 2,640 & 40 & 0 & 1 & & 2.4 \\
\hline 55 & 1 & 5 & 4 & 2,000 & 30 & 1 & 0 & Steep slopes & 1.4 \\
\hline 56 & $\mathrm{D}$ & 9 & 1 & 1,584 & 50 & 1 & 1 & Ditch work & 1.8 \\
\hline 57 & $9-22$ & 9 & 2 & 1,000 & 50 & 1 & 1 & $\begin{array}{l}\text { Construction } \\
\text { activity }\end{array}$ & 1.1 \\
\hline 58 & $9-22$ & 9 & 1 & 150 & 30 & 0 & 1 & Steep slope & 0.1 \\
\hline 59 & $9-22$ & 9 & 2 & 100 & 30 & 0 & 0 & Rd ruts & 0.1 \\
\hline 60 & $9-22$ & 9 & 2 & 200 & 30 & 0 & 1 & Rd ruts & 0.1 \\
\hline 61 & C-1.1 & 9 & 1 & 528 & 30 & 1 & 1 & Deep gullies & 0.4 \\
\hline 62 & C-1.1 & 9 & 4 & 2,640 & 30 & 0 & 1 & Ditch work & 1.8 \\
\hline 63 & East of 9-23 & 9 & 4 & 100 & 50 & 0 & 1 & Ditch work & 0.1 \\
\hline 64 & $\begin{array}{r}\text { Between C-1 } \\
\text { and D } \\
\end{array}$ & 9 & 4 & 1,000 & 30 & 0 & 1 & Ditch work & 0.7 \\
\hline 65 & Rd C-1.1 & 9 & 2 & 2,112 & 50 & 1 & 1 & Ditch work & 2.4 \\
\hline 66 & $9-31$ & 9 & 1 & 2,640 & 50 & 0 & 1 & Ditch work & 3.0 \\
\hline 67 & East of D & 9 & 4 & 300 & 40 & 1 & 0 & & 0.3 \\
\hline 68 & $9-32.1$ & 9 & 1 & 100 & 25 & 0 & 1 & Deep gully & 0.1 \\
\hline 69 & $9-32.1 \mathrm{a}$ & 9 & 4 & 1,584 & 40 & 0 & 1 & Ditch work & 1.5 \\
\hline 70 & $9-26$ & 9 & 2 & 7,392 & 40 & 1 & 1 & Ditch work & 6.8 \\
\hline 71 & NE of $9-27$ & 9 & 2 & 3,168 & 40 & 0 & 0 & Rd ruts & 2.9 \\
\hline 72 & 10 & 2 & 4 & 1,000 & 30 & 0 & 0 & & 0.7 \\
\hline 73 & 2 & 10 & 2 & 2,112 & 40 & 1 & 1 & Steep slopes & 1.9 \\
\hline 74 & $\begin{array}{r}\text { South of } 10- \\
24.3 \\
\end{array}$ & 10 & 4 & 1,584 & 30 & 0 & 1 & & 1.1 \\
\hline 75 & C-1 & 10 & 1 & 9,504 & 40 & 0 & 1 & $\begin{array}{l}\text { Steep slopes, } \\
\text { drainage }\end{array}$ & 8.7 \\
\hline
\end{tabular}




\begin{tabular}{|c|c|c|c|c|c|c|c|c|c|}
\hline $\begin{array}{l}\text { GIS } \\
\text { Code }\end{array}$ & Road Name & Comp. & Type & \begin{tabular}{|c|} 
Length \\
(ft)
\end{tabular} & $\begin{array}{c}\text { Width } \\
\text { (ft) }\end{array}$ & HP & Sed. & Remarks & Acres \\
\hline 76 & $10-24$ & 10 & 4 & 500 & 30 & 0 & 1 & & 0.3 \\
\hline 77 & Across SC 125 & 10 & 4 & 1,584 & 60 & 0 & 0 & & 2.2 \\
\hline 78 & SC 125 & 3 & 4 & 1,584 & 30 & 0 & 0 & & 1.1 \\
\hline 79 & $11-28$ & 11 & 2 & 5,280 & 40 & 0 & 0 & Unpassable & 4.8 \\
\hline 80 & C-1 & 11 & 1 & 1,000 & 40 & 0 & 0 & Ditch work & 0.9 \\
\hline 81 & C-1 & 11 & 2 & 2,640 & 40 & 0 & 1 & & 2.4 \\
\hline 82 & $\begin{array}{r}\text { Rds } 11-23,11- \\
30 \\
\end{array}$ & 11 & 4 & 1,056 & 40 & 0 & 1 & & 1.0 \\
\hline 83 & $\mathrm{Rd} 11-23$ & 11 & 1 & 2,112 & 40 & 0 & 1 & Ditch work & 1.9 \\
\hline 84 & C-2.1 & 13 & 1 & 1,584 & 40 & 1 & 1 & & 1.5 \\
\hline 85 & C-2.1 & 13 & 1 & 1,320 & 40 & 0 & 1 & Ditch work & 1.2 \\
\hline 86 & C-2.1 & 13 & 4 & 2,112 & 40 & 0 & 0 & & 1.9 \\
\hline 87 & $11-32$ & 11 & 2 & 2,112 & 30 & 0 & 1 & & 1.5 \\
\hline 88 & $11-22$ & 11 & 2 & 1,056 & 30 & 0 & 1 & & 0.7 \\
\hline 89 & $11-31$ & 11 & 2 & 3,696 & 40 & 0 & 0 & & 3.4 \\
\hline 90 & $\mathrm{C}$ & 13 & 4 & 4,224 & 30 & 0 & 1 & & 2.9 \\
\hline 91 & $13-27$ & 13 & 1 & 3,168 & 30 & 0 & 1 & $\begin{array}{l}\text { Ditch work; } \\
\text { sandy }\end{array}$ & 2.2 \\
\hline 92 & C-2.1 & 13 & 4 & 2,640 & 30 & 0 & 0 & & 1.8 \\
\hline 93 & East of C-2.1 & 13 & 4 & 1,056 & 30 & 0 & 0 & & 0.7 \\
\hline 94 & $\mathrm{Rd} 3$ & 14 & 1 & 550 & 80 & 0 & 1 & Steep slope & 1.0 \\
\hline 95 & $\mathrm{Rd} 3$ & 14 & 4 & 5,280 & 70 & 0 & 0 & & 8.5 \\
\hline 96 & $A-8$ & 14 & 1 & 0 & 0 & 1 & 0 & $\begin{array}{l}\text { Sand/soil } \\
\text { removal }\end{array}$ & 10.0 \\
\hline 97 & $14-20$ & 14 & 1 & 16,340 & 35 & 0 & 1 & Ditch work & 13.1 \\
\hline 98 & $14-21$ & 14 & 2 & 528 & 30 & 0 & 0 & Ruts & 0.4 \\
\hline 99 & A-7 & 14 & 1 & 200 & 40 & 0 & 1 & Ditch work & 0.2 \\
\hline 100 & A-8 & 14 & 4 & 4,224 & 40 & 0 & 1 & & 3.9 \\
\hline
\end{tabular}




\begin{tabular}{|c|c|c|c|c|c|c|c|c|c|}
\hline $\begin{array}{l}\text { GIS } \\
\text { Code }\end{array}$ & Road Name & Comp. & Type & \begin{tabular}{|c|} 
Length \\
(ft)
\end{tabular} & $\begin{array}{c}\text { Width } \\
\text { (ft) }\end{array}$ & HP & Sed. & Remarks & Acres \\
\hline 101 & Near 14-27.1 & 14 & 1 & 200 & 35 & 0 & 1 & Ditch work & 0.2 \\
\hline 102 & $\mathrm{~F}$ & 15 & 4 & 3,696 & 40 & 0 & 0 & & 3.4 \\
\hline 103 & $15-29$ & 15 & 1 & 100 & 40 & 0 & 1 & Ditch work & 0.1 \\
\hline 104 & $\mathrm{~F}-4$ & 15 & 1 & 400 & 40 & 0 & 1 & $\begin{array}{l}\text { Adjacent to pit; } \\
\text { perhaps } 25\end{array}$ & 0.4 \\
\hline 105 & $15-30$ & 15 & 4 & 1,000 & 40 & 0 & 1 & Rd ruts & 0.9 \\
\hline 106 & $15-23$ & 15 & 4 & 1,000 & 40 & 0 & 1 & & 0.9 \\
\hline 107 & $\mathrm{~F}-1$ & 15 & 2 & 5,280 & 35 & 0 & 1 & Ditch work & 4.2 \\
\hline 108 & $2-3$ & 15 & 4 & 3,696 & 40 & 0 & 0 & & 3.4 \\
\hline 109 & Into F-1 & 15 & 2 & 1,056 & 40 & 0 & 1 & & 1.0 \\
\hline 110 & $\mathrm{~F}-1$ & 15 & 2 & 4,224 & 40 & 0 & 0 & & 3.9 \\
\hline 111 & 2 & 9 & 4 & 70 & 40 & 0 & 0 & & 0.1 \\
\hline 112 & C-2 & 16 & 1 & 1,000 & 50 & 0 & 1 & $\begin{array}{l}\text { Steep slopes; } \\
\text { ditch work }\end{array}$ & 1.1 \\
\hline 113 & 2 & 16 & 1 & 1,800 & 80 & 0 & 0 & $\begin{array}{l}\text { Steep slope; } \\
\text { deep gullies }\end{array}$ & 3.3 \\
\hline 114 & $16-28$ & 16 & 4 & 800 & 40 & 0 & 1 & Rd unpassable & 0.7 \\
\hline 115 & 2 & 8 & 4 & 900 & 75 & 0 & 0 & & 1.6 \\
\hline 116 & $\mathrm{~F}$ & 16 & 4 & 5,280 & 40 & 0 & 0 & & 4.8 \\
\hline 117 & $\mathrm{~F}$ & 16 & 4 & 270 & 75 & 0 & 0 & Steep slopes & 0.5 \\
\hline 118 & $\mathrm{~F}$ & 16 & 1 & 800 & 70 & 0 & 1 & Ditch work; ruts & 1.3 \\
\hline 119 & $\mathrm{~F}-3$ & 15 & 1 & 10,560 & 70 & 0 & 1 & Deep gullies & 17.0 \\
\hline 120 & $16-21$ & 16 & 1 & 3,168 & 40 & 0 & 0 & Ditch work & 2.9 \\
\hline 121 & $\begin{array}{r}\text { F-1 (Greene } \\
\text { Rd) }\end{array}$ & 17 & 2 & 50 & 50 & 0 & 1 & Rd ruts & 0.1 \\
\hline 122 & $2-1$ & 17 & 2 & 13,200 & 70 & 0 & 0 & Ditch work & 21.2 \\
\hline 123 & $18-21$ & 18 & 2 & 1,000 & 30 & 0 & 1 & Ditch work & 0.7 \\
\hline 124 & $\mathrm{~F}-2$ & 18 & 1 & 250 & 40 & 0 & 0 & & 0.2 \\
\hline 125 & $2-1$ & 18 & 1 & 5,280 & 70 & 0 & 1 & Ditch work & 8.5 \\
\hline
\end{tabular}




\begin{tabular}{|c|c|c|c|c|c|c|c|c|c|}
\hline $\begin{array}{l}\text { GIS } \\
\text { Code }\end{array}$ & Road Name & Comp. & Type & \begin{tabular}{|c|} 
Length \\
(ft)
\end{tabular} & $\begin{array}{c}\text { Width } \\
\text { (ft) }\end{array}$ & HP & Sed. & Remarks & Acres \\
\hline 126 & $18-35$ to $F-4$ & 18 & 4 & 800 & 40 & 0 & 0 & & 0.7 \\
\hline 127 & $19-22$ & 19 & 2 & 1,000 & 60 & 0 & 1 & & 1.4 \\
\hline 128 & Above 2-1 & 19 & 4 & 1,000 & 60 & 0 & 0 & & 1.4 \\
\hline 129 & $20-24$ & 20 & 1 & 70 & 40 & 0 & 0 & Ditch work & 0.1 \\
\hline 130 & $19-24,19-26$ & 19 & 4 & 7,920 & 30 & 0 & 1 & & 5.5 \\
\hline 131 & $\begin{array}{l}\text { Treadway } \\
\text { Bridge Rd }\end{array}$ & 20 & 4 & 300 & 30 & 0 & 0 & & 0.2 \\
\hline 132 & US 278 & 20 & 3 & 350 & 20 & 0 & 1 & $\begin{array}{l}\text { Dirt piled along } \\
\mathrm{Rd}\end{array}$ & 0.2 \\
\hline 133 & US 278 & 20 & 4 & 1,200 & 30 & 0 & 0 & $\begin{array}{l}\text { Scattered spots } \\
\text { along } 278\end{array}$ & 0.8 \\
\hline 134 & US 278 & 21 & 3 & 75 & 10 & 0 & 1 & & 0.0 \\
\hline 135 & Phelps Rd & 23 & 1 & 400 & 25 & 0 & 1 & Includes dirt pile & 0.2 \\
\hline 136 & Phelps Rd & 23 & 1 & 290 & 60 & 0 & 1 & $\begin{array}{l}\text { Slopes; ditch } \\
\text { work }\end{array}$ & 0.4 \\
\hline 137 & 781.31 & 23 & 1 & 500 & 50 & 0 & 1 & $\begin{array}{l}\text { Unpassable; } \\
\text { ditch work. }\end{array}$ & 0.6 \\
\hline 138 & 781.2 & 21 & 1 & 5,280 & 50 & 0 & 1 & $\begin{array}{l}\text { Adjacent areas } \\
\text { require ditch }\end{array}$ & 6.1 \\
\hline 139 & $21-20$ & 21 & 1 & 1,500 & 30 & 0 & 1 & $\begin{array}{l}\text { One other } 10 x \\
50 \text { Type } 2 \text { strip }\end{array}$ & 1.0 \\
\hline 140 & 781.31 & 21 & 1 & 3,168 & 40 & 0 & 1 & $\begin{array}{l}1.05 \text { acres of } \\
\text { other inventory }\end{array}$ & 2.9 \\
\hline 141 & $21-20$ & 21 & 3 & 13,200 & 60 & 0 & 1 & & 18.2 \\
\hline 142 & $\begin{array}{r}\text { Above } \\
\text { Treadway }\end{array}$ & 22 & 3 & 250 & 60 & 0 & 0 & & 0.3 \\
\hline 143 & US 278 & 22 & 3 & 7,650 & 30 & 1 & 1 & Various areas & 5.3 \\
\hline 144 & US 278 & 22 & 3 & 7,650 & 30 & 1 & 1 & Various areas & 5.3 \\
\hline 145 & US 278 & 22 & 3 & 7,650 & 30 & 1 & 1 & Various areas & 5.3 \\
\hline 146 & US 278 & 23 & 3 & 7,650 & 30 & 1 & 1 & Various areas & 5.3 \\
\hline 147 & US 278 & 25 & 3 & 7,650 & 30 & 1 & 1 & Various areas & 5.3 \\
\hline 148 & $23-20$ & 23 & 1 & 200 & 30 & 0 & 1 & $\begin{array}{l}\text { One gully } 10 x \\
20\end{array}$ & 0.1 \\
\hline 149 & 781.2 & 23 & 3 & 2,640 & 50 & 0 & 1 & & 3.0 \\
\hline 150 & 23-20 & 23 & 3 & 700 & 40 & 0 & 1 & $\begin{array}{l}\text { One gully } 20 x \\
40\end{array}$ & 0.6 \\
\hline
\end{tabular}




\begin{tabular}{|c|c|c|c|c|c|c|c|c|c|}
\hline $\begin{array}{l}\text { GIS } \\
\text { Code }\end{array}$ & Road Name & Comp. & Type & $\begin{array}{l}\text { Length } \\
\text { (ft) }\end{array}$ & $\begin{array}{l}\text { Width } \\
\text { (ft) }\end{array}$ & HP & Sed. & Remarks & Acres \\
\hline 151 & By 23-21 & 23 & 3 & 550 & 80 & 0 & 1 & $\begin{array}{l}\text { Adjacent to farm } \\
\text { field }\end{array}$ & 1.0 \\
\hline 152 & $23-21$ & 23 & 3 & 13,200 & 50 & 0 & 1 & Type $120 \times 20$ & 15.2 \\
\hline 153 & 23-22 & 23 & 4 & 5,280 & 30 & 0 & 1 & $\begin{array}{l}\text { Unpassable Rd, } \\
\text { sandy. }\end{array}$ & 3.6 \\
\hline 154 & Cox Bridge Rd & 24 & 2 & 6,336 & 50 & 0 & 1 & & 7.3 \\
\hline 155 & 24-22 & 24 & 1 & 500 & 50 & 1 & 1 & & 0.6 \\
\hline 156 & Eubanks Rd & 24 & 4 & 2,000 & 30 & 0 & 0 & & 1.4 \\
\hline 157 & Eubanks Rd & 24 & 4 & 2,000 & 30 & 0 & 0 & & 1.4 \\
\hline 158 & Eubanks Rd & 24 & 4 & 2,000 & 30 & 0 & 0 & & 1.4 \\
\hline 159 & Eubanks Rd & 24 & 4 & 2,000 & 30 & 0 & 0 & & 1.4 \\
\hline 160 & Eubanks Rd & 24 & 4 & 2,000 & 30 & 0 & 0 & & 1.4 \\
\hline 161 & $24-23 \& 24$ & 24 & 2 & 300 & 30 & 0 & 1 & Rd ruts & 0.2 \\
\hline 162 & $\begin{array}{r}\text { Into Hickson } \\
\text { Mill Rd }\end{array}$ & 24 & 2 & 150 & 30 & 0 & 1 & & 0.1 \\
\hline 163 & Hickson Mill Rd & 25 & 3 & 2,640 & 30 & 0 & 1 & & 1.8 \\
\hline 164 & $25-22$ & 25 & 3 & 800 & 30 & 0 & 1 & & 0.6 \\
\hline 165 & $26-20$ & 26 & 3 & 5,600 & 30 & 0 & 1 & & 3.9 \\
\hline 166 & $\begin{array}{r}\text { Kennedy Pond } \\
\text { Rd }\end{array}$ & 26 & 2 & 75 & 20 & 0 & 0 & Rd ruts; washout & 0.0 \\
\hline 167 & $8-12$ & 27 & 4 & 500 & 30 & 0 & 1 & Washout & 0.3 \\
\hline 168 & $8-12$ & 27 & 4 & 400 & 30 & 0 & 1 & & 0.3 \\
\hline 169 & $\begin{array}{r}\text { Kennedy Pond } \\
\text { Rd }\end{array}$ & 27 & 2 & 50 & 30 & 0 & 1 & Washouts & 0.0 \\
\hline 170 & $28-24$ & 28 & 3 & 200 & 30 & 0 & 1 & Ditch work & 0.1 \\
\hline 171 & $28-20$ & 28 & 3 & 6,280 & 30 & 0 & 1 & Much sediment & 4.3 \\
\hline 172 & $28-21$ & 28 & 3 & 200 & 30 & 0 & 1 & Ditch work & 0.1 \\
\hline 173 & $28-21$ & 28 & 3 & 600 & 30 & 0 & 1 & Washouts & 0.4 \\
\hline 174 & 8 & 28 & 3 & 4,500 & 30 & 0 & 1 & & 3.1 \\
\hline 175 & $29-20$ & 29 & 3 & 2,200 & 30 & 0 & 1 & & 1.5 \\
\hline
\end{tabular}




\begin{tabular}{|c|c|c|c|c|c|c|c|c|c|}
\hline $\begin{array}{c}\text { GIS } \\
\text { Code }\end{array}$ & Road Name & Comp. & Type & \begin{tabular}{c|} 
Length \\
(ft)
\end{tabular} & $\begin{array}{l}\text { Width } \\
\text { (ft) }\end{array}$ & HP & Sed. & Remarks & Acres \\
\hline 176 & $29-20$ & 29 & 4 & 300 & 30 & 0 & 1 & Sandy & 0.2 \\
\hline 177 & 29-31 & 29 & 3 & 40 & 30 & 0 & 0 & Wetland over Rd & 0.0 \\
\hline 178 & $29-22.1 b$ & 29 & 3 & 50 & 30 & 0 & 1 & Sandy & 0.0 \\
\hline 179 & 8 & 29 & 3 & 2,740 & 30 & 0 & 1 & & 1.9 \\
\hline 180 & 8 & 30 & 3 & 2,740 & 30 & 0 & 1 & & 1.9 \\
\hline 181 & $30-22.2$ & 30 & 2 & 150 & 30 & 0 & 1 & Washouts & 0.1 \\
\hline 182 & $30-22$ & 30 & 3 & 30 & 30 & 0 & 0 & & 0.0 \\
\hline 183 & Into 31.24 .3 & 30 & 2 & 50 & 10 & 0 & 0 & Washouts & 0.0 \\
\hline 184 & $2-1$ & 33 & 1 & 220 & 35 & 0 & 1 & $\begin{array}{l}\text { Actually } 30 \times 220 \\
R, 30 \times 220 \mathrm{~L}\end{array}$ & 0.2 \\
\hline 185 & $32-26$ & 32 & 1 & 120 & 25 & 0 & 0 & $\begin{array}{l}\text { Ruts; } \\
\text { undriveable }\end{array}$ & 0.1 \\
\hline 186 & $2-1.1$ & 31 & 1 & 7,920 & 70 & 0 & 1 & Ditch work & 12.7 \\
\hline 187 & $32-24$ & 32 & 1 & 1,500 & 40 & 0 & 1 & Ditch work & 1.4 \\
\hline 188 & $\begin{array}{r}\text { Into Treadway } \\
\text { Bridge Rd }\end{array}$ & 32 & 1 & 1,000 & 25 & 0 & 1 & & 0.6 \\
\hline 189 & $\begin{array}{l}\text { Treadway } \\
\text { Bridge Rd } \\
\end{array}$ & 32 & 1 & 1,500 & 35 & 0 & 1 & Steep slopes & 1.2 \\
\hline 190 & $2-1$ & 31 & 1 & 400 & 25 & 0 & 0 & Ditch work & 0.2 \\
\hline 191 & $33-20$ & 33 & 1 & 1,584 & 40 & 0 & 0 & Logging activity & 1.5 \\
\hline 192 & $33-20$ & 33 & 2 & 1,584 & 40 & 0 & 0 & & 1.5 \\
\hline 193 & $33-20$ & 33 & 2 & 250 & 30 & 0 & 1 & & 0.2 \\
\hline 194 & $33-20$ & 33 & 4 & 400 & 30 & 0 & 1 & & 0.3 \\
\hline 195 & $\begin{array}{r}\text { Ahead Parks } \\
\text { Church Rd }\end{array}$ & 35 & 3 & 30 & 20 & 0 & 1 & $\begin{array}{l}\text { Sediment into } \\
\text { creek }\end{array}$ & 0.0 \\
\hline 196 & $36-27.1$ & 36 & 3 & 100 & 30 & 0 & 1 & & 0.1 \\
\hline 197 & $38-28$ & 38 & 3 & 1,320 & 40 & 0 & 0 & & 1.2 \\
\hline 198 & $38-28.1$ & 38 & 2 & 25 & 20 & 0 & 1 & Washouts & 0.0 \\
\hline 199 & $38-24$ & 38 & 4 & 35 & 30 & 0 & 1 & & 0.0 \\
\hline 200 & $38-24$ & 38 & 3 & 100 & 40 & 0 & 0 & & 0.1 \\
\hline
\end{tabular}


1991 SRS Soil Erosion and Sediemnt Results

\begin{tabular}{|c|c|c|c|c|c|c|c|c|c|}
\hline $\begin{array}{l}\text { GIS } \\
\text { Code }\end{array}$ & Road Name & Comp. & Type & \begin{tabular}{|c|} 
Length \\
(ft)
\end{tabular} & $\begin{array}{c}\text { Width } \\
\text { (ft) }\end{array}$ & HP & Sed. & Remarks & Acres \\
\hline 201 & $38-22.1$ & 38 & 1 & 20 & 30 & 0 & 1 & & 0.0 \\
\hline 202 & $38-22$ & 38 & 3 & 1,584 & 40 & 0 & 1 & & 1.5 \\
\hline 203 & $38-29$ & 38 & 3 & 6,336 & 40 & 0 & 1 & & 5.8 \\
\hline 204 & $38-22$ & 38 & 3 & 9,504 & 40 & 0 & 1 & & 8.7 \\
\hline 205 & $\begin{array}{r}\text { East of } 38- \\
22.4 a \\
\end{array}$ & 38 & 3 & 9,504 & 40 & 0 & 1 & & 8.7 \\
\hline 206 & $38-22$ & 38 & 2 & 1,584 & 40 & 0 & 1 & & 1.5 \\
\hline 207 & $38-21.1$ & 38 & 3 & 5,280 & 40 & 0 & 1 & & 4.8 \\
\hline 208 & North of $38-27$ & 38 & 1 & 250 & 50 & 0 & 0 & & 0.3 \\
\hline 209 & Robbins Rd & 38 & 3 & 2,640 & 40 & 0 & 1 & & 2.4 \\
\hline 210 & $38-33.1$ & 38 & 3 & 3,168 & 50 & 0 & 0 & $\begin{array}{l}\text { One } 10 \text { x } 20 \\
\text { gully }\end{array}$ & 3.6 \\
\hline 211 & SC 125 & 38 & 3 & 400 & 10 & 0 & 1 & $\begin{array}{l}\text { Many small } \\
\text { areas }\end{array}$ & 0.1 \\
\hline 212 & SC 125 & 38 & 3 & 400 & 10 & 0 & 1 & $\begin{array}{l}\text { Many small } \\
\text { areas }\end{array}$ & 0.1 \\
\hline 213 & SC 125 & 38 & 3 & 400 & 10 & 0 & 1 & $\begin{array}{l}\text { Many small } \\
\text { areas }\end{array}$ & 0.1 \\
\hline 214 & SC 125 & 38 & 3 & 400 & 10 & 0 & 1 & $\begin{array}{l}\text { Many small } \\
\text { areas }\end{array}$ & 0.1 \\
\hline 215 & Station $\mathrm{Rd}$ & 38 & 3 & 9,504 & 50 & 0 & 0 & & 10.9 \\
\hline 216 & $39-20$ & 39 & 3 & 2,112 & 40 & 0 & 1 & Sandy & 1.9 \\
\hline 217 & $39-20$ & 39 & 4 & 100 & 30 & 0 & 0 & & 0.1 \\
\hline 218 & $40-39$ & 40 & 2 & 100 & 30 & 0 & 1 & & 0.1 \\
\hline 219 & $40-39$ & 40 & 3 & 5,280 & 30 & 0 & 1 & Sandy & 3.6 \\
\hline 220 & $40-39$ & 40 & 1 & 60 & 10 & 0 & 0 & Washouts & 0.0 \\
\hline 221 & B-5 & 40 & 2 & 2,640 & 40 & 0 & 1 & & 2.4 \\
\hline 222 & 9 & 41 & 3 & 100 & 20 & 0 & 1 & & 0.0 \\
\hline 223 & Meyers Mill Rd & 40 & 3 & 30 & 10 & 0 & 0 & & 0.0 \\
\hline 224 & $41-21$ & 41 & 3 & 5,280 & 40 & 0 & 1 & New Rd & 4.8 \\
\hline 225 & $41-28$ & 41 & 4 & 100 & 10 & 0 & 1 & & 0.0 \\
\hline
\end{tabular}


1991 SRS Soil Erosion and Sediemnt Results

\begin{tabular}{|c|c|c|c|c|c|c|c|c|c|}
\hline $\begin{array}{l}\text { GIS } \\
\text { Code }\end{array}$ & Road Name & Comp. & Type & \begin{tabular}{|c|} 
Length \\
(ft)
\end{tabular} & $\begin{array}{l}\text { Width } \\
\text { (ft) }\end{array}$ & HP & Sed. & Remarks & Acres \\
\hline 226 & $41-20$ & 41 & 3 & 65 & 15 & 0 & 1 & & 0.0 \\
\hline 227 & West of $41-29$ & 41 & 4 & 100 & 30 & 1 & 0 & & 0.1 \\
\hline 228 & Trestle Rd & 41 & 3 & 500 & 40 & 0 & 1 & & 0.5 \\
\hline 229 & $41-22$ & 41 & 3 & 75 & 20 & 0 & 1 & & 0.0 \\
\hline 230 & $42-28$ & 42 & 2 & 100 & 30 & 0 & 0 & & 0.1 \\
\hline 231 & A-13.1 & 42 & 2 & 50 & 20 & 0 & 0 & & 0.0 \\
\hline 232 & $42-25$ & 42 & 1 & 600 & 30 & 0 & 1 & Deep gully & 0.4 \\
\hline 233 & SC 125 & 43 & 4 & 125 & 30 & 0 & 0 & Steep slopes & 0.1 \\
\hline 234 & SC 125 & 43 & 4 & 500 & 40 & 1 & 1 & Steep slope & 0.5 \\
\hline 235 & $\begin{array}{l}\text { Near Comp. } \\
\text { 42/39 Border }\end{array}$ & 39 & 1 & 600 & 30 & 0 & 0 & & 0.4 \\
\hline 236 & SC 125 & 43 & 4 & 100 & 30 & 0 & 0 & & 0.1 \\
\hline 237 & $\mathrm{~B}$ & 43 & 4 & 300 & 30 & 0 & 1 & & 0.2 \\
\hline 238 & $38-34.3$ & 45 & 2 & 100 & 50 & 0 & 0 & & 0.1 \\
\hline 239 & East of $45-34$ & 45 & 4 & 2,112 & 30 & 0 & 1 & Washouts & 1.5 \\
\hline 240 & $45-24$ & 45 & 3 & 1,028 & 30 & 0 & 0 & & 0.7 \\
\hline 241 & $46-23$ & 46 & 2 & 200 & 30 & 0 & 1 & & 0.1 \\
\hline 242 & $47-25$ & 47 & 2 & 50 & 30 & 0 & 1 & & 0.0 \\
\hline 243 & East of $47-25$ & 47 & 2 & 50 & 30 & 0 & 1 & & 0.0 \\
\hline 244 & A-11 & 47 & 3 & 50 & 40 & 0 & 0 & & 0.0 \\
\hline 245 & A-9 & 47 & 3 & 1,056 & 40 & 0 & 1 & & 1.0 \\
\hline 246 & 3 & 48 & 1 & 1,584 & 40 & 0 & 1 & & 1.5 \\
\hline 247 & Burma Rd & 48 & 1 & 6,336 & 70 & 0 & 1 & & 10.2 \\
\hline 248 & $A-6$ & 48 & 4 & 800 & 40 & 0 & 1 & & 0.7 \\
\hline 249 & West of $48-27$ & 48 & 1 & 0 & 0 & 0 & 1 & $\begin{array}{l}\text { New construction } \\
\text { pit }\end{array}$ & 40.0 \\
\hline 250 & A-7 & 48 & 1 & 2,112 & 65 & 0 & 1 & & 3.2 \\
\hline
\end{tabular}


1991 SRS Soil Erosion and Sediemnt Results

\begin{tabular}{|c|c|c|c|c|c|c|c|c|c|}
\hline $\begin{array}{c}\text { GIS } \\
\text { Code }\end{array}$ & Road Name & Comp. & Type & $\begin{array}{c}\text { Length } \\
\text { (ft) }\end{array}$ & $\begin{array}{c}\text { Width } \\
\text { (ft) }\end{array}$ & HP & Sed. & Remarks & Acres \\
\hline 251 & $A-7$ & 48 & 1 & 1,584 & 40 & 0 & 0 & & 1.5 \\
\hline 252 & $A-6$ & 48 & 1 & 1,584 & 30 & 0 & 1 & & 1.1 \\
\hline 253 & $\mathrm{C}$ & 49 & 4 & 200 & 60 & 0 & 1 & & 0.3 \\
\hline 254 & $\mathrm{C}$ & 49 & 2 & 350 & 60 & 0 & 1 & & 0.5 \\
\hline 255 & 4 & 49 & 2 & 450 & 30 & 0 & 0 & Steep slopes & 0.3 \\
\hline 256 & 4 & 49 & 2 & 2,200 & 30 & 0 & 1 & & 1.5 \\
\hline 257 & 4 & 49 & 4 & 60 & 25 & 0 & 0 & & 0.0 \\
\hline 258 & $49-21$ & 49 & 2 & 20 & 30 & 0 & 1 & & 0.0 \\
\hline 259 & West of $49-21$ & 49 & 1 & 8,045 & 40 & 1 & 0 & $\begin{array}{l}\text { Near a 15-acre } \\
\text { bare area }\end{array}$ & 7.4 \\
\hline 260 & $49-31$ & 49 & 4 & 0 & 0 & 0 & 0 & $\begin{array}{l}2 \text { acres near } \\
\text { large area }\end{array}$ & 2.0 \\
\hline 261 & $E$ & 49 & 1 & 2,112 & 30 & 0 & 1 & & 1.5 \\
\hline 262 & 4 & 50 & 3 & 650 & 20 & 0 & 1 & & 0.3 \\
\hline 263 & $50-36$ & 50 & 1 & 500 & 30 & 0 & 1 & Gulley & 0.3 \\
\hline 264 & $50-37.2$ & 50 & 1 & 1,800 & 40 & 0 & 1 & Washouts & 1.7 \\
\hline 265 & 4 & 50 & 2 & 800 & 10 & 0 & 0 & $\begin{array}{l}\text { Sediment flowing } \\
\text { into creek }\end{array}$ & 0.2 \\
\hline 266 & $50-34$ & 50 & 2 & 35 & 30 & 0 & 1 & & 0.0 \\
\hline 267 & South of $50-31$ & 50 & 2 & 90 & 30 & 0 & 1 & $\begin{array}{l}\text { Wetland flows } \\
\text { over Rd }\end{array}$ & 0.1 \\
\hline 268 & $50-35$ & 50 & 1 & 50 & 50 & 0 & 1 & $\begin{array}{l}\text { Sediment } \\
\text { deposits into }\end{array}$ & 0.1 \\
\hline 269 & $50-29$ & 50 & 4 & 100 & 20 & 0 & 1 & & 0.0 \\
\hline 270 & North of $50-26$ & 50 & 1 & 230 & 10 & 0 & 1 & Washouts & 0.1 \\
\hline 271 & $50-26$ & 50 & 4 & 450 & 20 & 0 & 0 & & 0.2 \\
\hline 272 & $\begin{array}{r}\text { Intersects with } \\
50-28.1\end{array}$ & 50 & 2 & 100 & 10 & 0 & 1 & $\begin{array}{l}\text { Four more acres } \\
\text { eroding }\end{array}$ & 0.0 \\
\hline 273 & $50-39$ & 50 & 2 & 1,584 & 20 & 0 & 1 & & 0.7 \\
\hline 274 & $50-39$ & 50 & 2 & 150 & 40 & 0 & 1 & & 0.1 \\
\hline 275 & $50-39$ & 50 & 4 & 20 & 10 & 0 & 1 & & 0.0 \\
\hline
\end{tabular}




\begin{tabular}{|c|c|c|c|c|c|c|c|c|c|}
\hline $\begin{array}{c}\text { GIS } \\
\text { Code }\end{array}$ & Road Name & Comp. & Type & \begin{tabular}{|c|} 
Length \\
(ft)
\end{tabular} & $\begin{array}{c}\text { Width } \\
\text { (ft) }\end{array}$ & HP & Sed. & Remarks & Acres \\
\hline 276 & $50-39$ & 50 & 4 & 500 & 40 & 0 & 1 & & 0.5 \\
\hline 277 & $50-23$ & 50 & 3 & 50 & 20 & 0 & 0 & & 0.0 \\
\hline 278 & $50-23$ & 50 & 4 & 50 & 30 & 0 & 0 & & 0.0 \\
\hline 279 & Southeast of $F$ & 50 & 1 & 200 & 50 & 0 & 1 & & 0.2 \\
\hline 280 & $\begin{array}{r}\text { Z-Area } \\
\text { Perimeter }\end{array}$ & 51 & 1 & 3,300 & 40 & 0 & 1 & & 3.0 \\
\hline 281 & 51-33.3 & 51 & 1 & 700 & 12 & 0 & 1 & & 0.2 \\
\hline 282 & 51-34.2 & 51 & 1 & 5,280 & 40 & 0 & 1 & & 4.8 \\
\hline 283 & Into 51-39 & 51 & 1 & 140 & 30 & 0 & 1 & & 0.1 \\
\hline 284 & $\begin{array}{r}\text { Monroe Owens } \\
\mathrm{Rd}\end{array}$ & 51 & 2 & 1,000 & 50 & 0 & 0 & & 1.1 \\
\hline 285 & Across 51-38 & 51 & 4 & 60 & 30 & 0 & 1 & & 0.0 \\
\hline 286 & $51-35$ & 51 & 1 & 5,280 & 50 & 0 & 0 & & 6.1 \\
\hline 287 & Beaufort Rd & 52 & 4 & 120 & 30 & 0 & 0 & & 0.1 \\
\hline 288 & East of $51-36$ & 51 & 4 & 400 & 40 & 0 & 0 & & 0.4 \\
\hline 289 & $\mathrm{~F}$ & 50 & 4 & 800 & 30 & 0 & 0 & & 0.6 \\
\hline 290 & $51-26$ & 51 & 1 & 1,600 & 50 & 0 & 1 & Stream crossing & 1.8 \\
\hline 291 & $51-33$ & 51 & 1 & 1,200 & 60 & 0 & 1 & $\begin{array}{l}\text { Spoil pile; Rd } \\
\text { side gullies }\end{array}$ & 1.7 \\
\hline 292 & $\begin{array}{r}\text { Monroe Owens } \\
\mathrm{Rd}\end{array}$ & 51 & 1 & 12,144 & 60 & 0 & 1 & & 16.7 \\
\hline 293 & Beaufort Rd & 51 & 1 & 1,584 & 70 & 0 & 0 & & 2.5 \\
\hline 294 & $\begin{array}{r}\text { Runs into } \\
\text { Monroe Owens } \\
\end{array}$ & 51 & 4 & 528 & 30 & 0 & 0 & & 0.4 \\
\hline 295 & Runs into Rd E & 52 & 1 & 150 & 30 & 0 & 0 & Deep gullies & 0.1 \\
\hline 296 & $\begin{array}{r}\text { Runs into } \\
\text { Monroe Owens } \\
\end{array}$ & 52 & 3 & 100 & 30 & 0 & 0 & & 0.1 \\
\hline 297 & $\begin{array}{r}\text { Monroe Owens } \\
\mathrm{Rd}\end{array}$ & 52 & 2 & 220 & 70 & 0 & 1 & & 0.4 \\
\hline 298 & $\begin{array}{r}\text { Monroe Owens } \\
\mathrm{Rd}\end{array}$ & 52 & 1 & 5,280 & 70 & 0 & 0 & & 8.5 \\
\hline 299 & $52-30.1$ & 52 & 1 & 350 & 70 & 0 & 1 & Ditch work & 0.6 \\
\hline 300 & $52-33$ & 52 & 1 & 2,640 & 70 & 0 & 1 & & 4.2 \\
\hline
\end{tabular}




\begin{tabular}{|c|c|c|c|c|c|c|c|c|c|}
\hline $\begin{array}{l}\text { GIS } \\
\text { Code }\end{array}$ & Road Name & Comp. & Type & $\begin{array}{c}\text { Length } \\
\text { (ft) }\end{array}$ & $\begin{array}{l}\text { Width } \\
\text { (ft) }\end{array}$ & HP & Sed. & Remarks & Acres \\
\hline 301 & Woodward Rd & 53 & 0 & 50 & 40 & 0 & 0 & $\begin{array}{l}\text { Wetland across } \\
\text { Rd }\end{array}$ & 0.0 \\
\hline 302 & $8-8$ & 53 & 0 & 300 & 30 & 0 & 0 & $\begin{array}{l}\text { Wetland across } \\
\text { Rd }\end{array}$ & 0.2 \\
\hline 303 & Woodward Rd & 53 & 2 & 80 & 40 & 0 & 0 & & 0.1 \\
\hline 304 & $\begin{array}{r}\text { Monroe Owens } \\
\mathrm{Rd}\end{array}$ & 53 & 1 & 300 & 70 & 0 & 1 & & 0.5 \\
\hline 305 & Pleasant Hill Rd & 54 & 4 & 200 & 40 & 0 & 1 & & 0.2 \\
\hline 306 & Pleasant Hill Rd & 54 & 4 & 100 & 40 & 0 & 1 & & 0.1 \\
\hline 307 & 8 & 54 & 4 & 100 & 40 & 0 & 0 & & 0.1 \\
\hline 308 & Craig Rd & 54 & 4 & 1,584 & 30 & 0 & 1 & & 1.1 \\
\hline 309 & Craig Rd & 54 & 4 & 600 & 30 & 0 & 0 & & 0.4 \\
\hline 310 & 8 & 54 & 4 & 300 & 40 & 0 & 1 & & 0.3 \\
\hline 311 & $56-29.1$ & 56 & 2 & 125 & 25 & 0 & 1 & & 0.1 \\
\hline 312 & 8 & 57 & 2 & 500 & 60 & 0 & 0 & & 0.7 \\
\hline 313 & 8 & 57 & 4 & 550 & 30 & 0 & 1 & & 0.4 \\
\hline 314 & $57-21.1$ & 57 & 3 & 500 & 30 & 0 & 1 & Sandy & 0.3 \\
\hline 315 & $5-21$ & 57 & 4 & 50 & 30 & 0 & 1 & & 0.0 \\
\hline 316 & $57-20$ & 57 & 4 & 800 & 30 & 0 & 1 & Ruts; washouts & 0.6 \\
\hline 317 & $\mathrm{G}$ & 58 & 3 & 2,500 & 60 & 0 & 0 & & 3.4 \\
\hline 318 & $\mathrm{G}$ & 58 & 3 & 650 & 30 & 0 & 1 & Steep slope & 0.4 \\
\hline 319 & $58-22$ & 58 & 3 & 600 & 30 & 0 & 1 & & 0.4 \\
\hline 320 & $58-22$ & 58 & 3 & 50 & 30 & 0 & 0 & & 0.0 \\
\hline 321 & $58-20.2$ & 58 & 3 & 200 & 30 & 0 & 1 & & 0.1 \\
\hline 322 & Runs into 58-33 & 58 & 3 & 30 & 10 & 0 & 0 & & 0.0 \\
\hline 323 & South of $58-20$ & 58 & 4 & 1,000 & 30 & 0 & 1 & Washouts & 0.7 \\
\hline 324 & $58-26$ & 58 & 1 & 1,600 & 30 & 0 & 1 & $\begin{array}{l}\text { Mostly bare soils; } \\
\text { one } 15 \times 50 \text { gully }\end{array}$ & 1.1 \\
\hline 325 & East of $58-26$ & 58 & 3 & 50 & 30 & 0 & 1 & & 0.0 \\
\hline
\end{tabular}




\begin{tabular}{|c|c|c|c|c|c|c|c|c|c|}
\hline $\begin{array}{c}\text { GIS } \\
\text { Code }\end{array}$ & Road Name & Comp. & Type & \begin{tabular}{c|} 
Length \\
(ft)
\end{tabular} & $\begin{array}{l}\text { Width } \\
\text { (ft) }\end{array}$ & HP & Sed. & Remarks & Acres \\
\hline 326 & $58-26$ & 58 & 3 & 5,280 & 30 & 0 & 1 & Sandy & 3.6 \\
\hline 327 & 58-30 & 58 & 3 & 50 & 30 & 0 & 1 & Washouts & 0.0 \\
\hline 328 & $\begin{array}{r}\text { Monroe Owens } \\
\mathrm{Rd} \\
\end{array}$ & 58 & 3 & 500 & 30 & 0 & 0 & & 0.3 \\
\hline 329 & $53-20$ & 53 & 3 & 400 & 30 & 0 & 0 & & 0.3 \\
\hline 330 & $53-20$ & 53 & 4 & 200 & 30 & 0 & 1 & $\begin{array}{l}\text { Sandy; wetland } \\
\text { over Rd }\end{array}$ & 0.1 \\
\hline 331 & $58-35$ & 58 & 3 & 350 & 30 & 0 & 1 & & 0.2 \\
\hline 332 & Beaufort Rd & 60 & 2 & 250 & 50 & 0 & 0 & & 0.3 \\
\hline 333 & Off of $77-20.3 a$ & 77 & 3 & 7,920 & 40 & 0 & 1 & Washouts & 7.3 \\
\hline 334 & $61-23.1(1 b)$ & 61 & 3 & 2,112 & 30 & 0 & 1 & & 1.5 \\
\hline 335 & $61-23$ & 61 & 4 & 1,056 & 30 & 0 & 1 & & 0.7 \\
\hline 336 & 63-36 & 63 & 3 & 35 & 10 & 0 & 0 & & 0.0 \\
\hline 337 & By $63-36$ & 63 & 1 & 528 & 40 & 0 & 1 & $\begin{array}{l}\text { Mostly type } 3 \\
\text { and 4; one } 10 x\end{array}$ & 0.5 \\
\hline 338 & $61-37.2 a$ & 61 & 3 & 50 & 30 & 0 & 1 & & 0.0 \\
\hline 339 & South of $61-23$ & 61 & 1 & 100 & 40 & 0 & 1 & & 0.1 \\
\hline 340 & 63-36 & 63 & 2 & 400 & 50 & 0 & 1 & & 0.5 \\
\hline 341 & $61-37.4$ & 61 & 3 & 3,168 & 30 & 0 & 1 & Very sandy & 2.2 \\
\hline 342 & B & 62 & 4 & 2,640 & 30 & 0 & 0 & $\begin{array}{l}\text { Construction } \\
\text { work at dam }\end{array}$ & 1.8 \\
\hline 343 & North of $62-31$ & 62 & 3 & 150 & 100 & 1 & 0 & & 0.3 \\
\hline 344 & $62-29$ & 62 & 3 & 35 & 10 & 0 & 0 & & 0.0 \\
\hline 345 & 63-31.6 & 63 & 2 & 6,336 & 5 & 0 & 1 & & 0.7 \\
\hline 346 & 63-31 & 63 & 1 & 550 & 30 & 0 & 0 & $\begin{array}{l}\text { Mostly sheet } \\
\text { erosion; one } 20 x\end{array}$ & 0.4 \\
\hline 347 & 63-34.1 & 63 & 2 & 50 & 20 & 0 & 1 & & 0.0 \\
\hline 348 & 7 & 63 & 2 & 500 & 50 & 0 & 0 & & 0.6 \\
\hline 349 & $\mathrm{~F}$ & 63 & 3 & 70 & 30 & 0 & 0 & & 0.0 \\
\hline 350 & $58-31$ & 58 & 3 & 50 & 25 & 0 & 0 & & 0.0 \\
\hline
\end{tabular}


1991 SRS Soil Erosion and Sediemnt Results

\begin{tabular}{|c|c|c|c|c|c|c|c|c|c|}
\hline $\begin{array}{c}\text { GIS } \\
\text { Code }\end{array}$ & Road Name & Comp. & Type & $\begin{array}{l}\text { Length } \\
\text { (ft) }\end{array}$ & $\begin{array}{c}\text { Width } \\
\text { (ft) }\end{array}$ & HP & Sed. & Remarks & Acres \\
\hline 351 & $E$ & 64 & 3 & 30 & 30 & 0 & 0 & & 0.0 \\
\hline 352 & 6 & 64 & 3 & 1,150 & 20 & 0 & 0 & & 0.5 \\
\hline 353 & 6 & 64 & 4 & 200 & 20 & 0 & 0 & & 0.1 \\
\hline 354 & 6 & 64 & 3 & 800 & 30 & 0 & 0 & & 0.6 \\
\hline 355 & $F$ and 6 & 64 & 3 & 0 & 0 & 0 & 0 & & 0.1 \\
\hline 356 & F-6 & 64 & 2 & 1,000 & 30 & 0 & 0 & & 0.7 \\
\hline 357 & $65-20$ & 65 & 2 & 550 & 30 & 0 & 0 & & 0.4 \\
\hline 358 & $E$ & 65 & 4 & 1,000 & 40 & 0 & 0 & & 0.9 \\
\hline 359 & Old House Rd & 65 & 1 & 1,056 & 40 & 0 & 1 & & 1.0 \\
\hline 360 & $E-1$ & 65 & 1 & 4,224 & 40 & 0 & 1 & & 3.9 \\
\hline 361 & $65-34$ & 65 & 1 & 3,696 & 40 & 0 & 1 & & 3.4 \\
\hline 362 & 6 & 66 & 4 & 1,200 & 100 & 0 & 0 & $\begin{array}{l}\text { Four } 100 \times 300 \\
\text { strips }\end{array}$ & 2.8 \\
\hline 363 & $\mathrm{~F}$ & 66 & 4 & 450 & 10 & 0 & 0 & & 0.1 \\
\hline 364 & Old House Rd & 65 & 4 & 40 & 10 & 0 & 0 & $\begin{array}{l}\text { New culvert over } \\
\text { stream }\end{array}$ & 0.0 \\
\hline 365 & Old House Rd & 65 & 1 & 130 & 50 & 0 & 0 & & 0.1 \\
\hline 366 & C & 66 & 4 & 208 & 208 & 0 & 0 & $\begin{array}{l}\text { Actually } 1 \text { acre } \\
\text { area }\end{array}$ & 1.0 \\
\hline 367 & 6 & 66 & 4 & 350 & 30 & 0 & 0 & $\begin{array}{l}\text { Two } 30 \times 175 \\
\text { strips }\end{array}$ & 0.2 \\
\hline 368 & 6 & 66 & 1 & 600 & 40 & 0 & 0 & & 0.6 \\
\hline 369 & $\mathrm{C}$ & 68 & 2 & 150 & 30 & 0 & 0 & Two areas & 0.1 \\
\hline 370 & Off Rd 3 & 66 & 1 & 600 & 40 & 0 & 1 & & 0.6 \\
\hline 371 & $66-25$ & 66 & 1 & 110 & 10 & 0 & 1 & & 0.0 \\
\hline 372 & $66-25$ & 66 & 4 & 2,640 & 50 & 0 & 1 & & 3.0 \\
\hline 373 & $66-25$ & 66 & 1 & 10 & 20 & 0 & 0 & & 0.0 \\
\hline 374 & $E-1$ & 66 & 1 & 540 & 30 & 0 & 0 & & 0.4 \\
\hline 375 & C-5 & 66 & 1 & 1,100 & 60 & 0 & 1 & & 1.5 \\
\hline
\end{tabular}




\begin{tabular}{|c|c|c|c|c|c|c|c|c|c|}
\hline $\begin{array}{l}\text { GIS } \\
\text { Code }\end{array}$ & Road Name & Comp. & Type & \begin{tabular}{|c|} 
Length \\
(ft)
\end{tabular} & $\begin{array}{c}\text { Width } \\
\text { (ft) }\end{array}$ & HP & Sed. & Remarks & Acres \\
\hline 376 & $C-5$ & 66 & 1 & 800 & 15 & 0 & 0 & & 0.3 \\
\hline 377 & $F-6$ & 66 & 1 & 375 & 60 & 0 & 1 & & 0.5 \\
\hline 378 & Youman Rd & 66 & 4 & 650 & 50 & 0 & 1 & & 0.7 \\
\hline 379 & $E-1$ & 66 & 4 & 100 & 20 & 0 & 1 & Sandy; two areas & 0.0 \\
\hline 380 & $E-1$ & 66 & 1 & 1,500 & 60 & 0 & 1 & & 2.1 \\
\hline 381 & E-1 & 66 & 1 & 3,168 & 60 & 0 & 1 & Wetland & 4.4 \\
\hline 382 & $\begin{array}{r}\text { North of } 67- \\
20.5 \\
\end{array}$ & 67 & 4 & 2,640 & 30 & 0 & 1 & & 1.8 \\
\hline 383 & $67-20.5$ & 67 & 2 & 125 & 30 & 0 & 1 & & 0.1 \\
\hline 384 & Off $67-40$ & 67 & 3 & 500 & 40 & 0 & 0 & & 0.5 \\
\hline 385 & Burma Rd & 67 & 3 & 1,000 & 40 & 0 & 1 & & 0.9 \\
\hline 386 & $67-21$ & 67 & 2 & 200 & 30 & 0 & 1 & & 0.1 \\
\hline 387 & C & 65 & 3 & 2,112 & 70 & 0 & 1 & & 3.4 \\
\hline 388 & $C-5$ & 67 & 4 & 2,640 & 45 & 0 & 1 & & 2.7 \\
\hline 389 & 5 & 67 & 4 & 160 & 12 & 0 & 0 & Steep slope & 0.0 \\
\hline 390 & $C-5$ & 67 & 2 & 1,584 & 40 & 0 & 1 & & 1.5 \\
\hline 391 & 99 & 68 & 4 & 50 & 10 & 0 & 0 & & 0.0 \\
\hline 392 & 68-38 & 68 & 3 & 300 & 30 & 0 & 0 & & 0.2 \\
\hline 393 & Above $\mathrm{Rd} 3$ & 68 & 3 & 500 & 40 & 0 & 0 & & 0.5 \\
\hline 394 & $68-31$ & 68 & 3 & 1,000 & 40 & 0 & 0 & & 0.9 \\
\hline 395 & A-7 & 68 & 2 & 200 & 30 & 0 & 0 & & 0.1 \\
\hline 396 & $68-34$ & 68 & 3 & 2,112 & 70 & 0 & 1 & & 3.4 \\
\hline 397 & $C-6$ & 69 & 2 & 700 & 40 & 0 & 1 & $\begin{array}{l}\text { Sediment into } \\
\text { creek }\end{array}$ & 0.6 \\
\hline 398 & Above 69-31.2 & 69 & 2 & 420 & 40 & 0 & 0 & & 0.4 \\
\hline 399 & Near 69-31.2 & 69 & 1 & 50 & 20 & 0 & 1 & & 0.0 \\
\hline 400 & Youman Rd & 69 & 2 & 550 & 40 & 0 & 1 & Sandy & 0.5 \\
\hline
\end{tabular}




\begin{tabular}{|c|c|c|c|c|c|c|c|c|c|}
\hline $\begin{array}{l}\text { GIS } \\
\text { Code }\end{array}$ & Road Name & Comp. & Type & \begin{tabular}{|c|} 
Length \\
(ft)
\end{tabular} & $\begin{array}{c}\text { Width } \\
\text { (ft) }\end{array}$ & HP & Sed. & Remarks & Acres \\
\hline 401 & $\mathrm{C}$ & 69 & 3 & 50 & 10 & 0 & 0 & High walls & 0.0 \\
\hline 402 & Williams Rd & 69 & 3 & 40 & 40 & 0 & 1 & $\begin{array}{l}\text { Sediment into } \\
\text { stream }\end{array}$ & 0.0 \\
\hline 403 & Youman Rd & 69 & 2 & 200 & 40 & 0 & 1 & & 0.2 \\
\hline 404 & 7 & 69 & 2 & 225 & 30 & 0 & 0 & & 0.2 \\
\hline 405 & $74-37.1$ & 69 & 1 & 210 & 30 & 0 & 1 & & 0.1 \\
\hline 406 & 6 & 70 & 2 & 100 & 50 & 1 & 1 & & 0.1 \\
\hline 407 & Youman Rd & 70 & 1 & 1,848 & 50 & 0 & 1 & & 2.1 \\
\hline 408 & $70-26$ & 70 & 2 & 100 & 50 & 0 & 1 & & 0.1 \\
\hline 409 & $70-30$ & 70 & 2 & 50 & 30 & 0 & 1 & & 0.0 \\
\hline 410 & $70-30$ & 70 & 4 & 50 & 30 & 0 & 1 & & 0.0 \\
\hline 411 & 517 & 70 & 4 & 100 & 50 & 0 & 0 & & 0.1 \\
\hline 412 & Across 73-33 & 70 & 2 & 65 & 30 & 1 & 1 & & 0.0 \\
\hline 413 & $\mathrm{~B}$ & 71 & 4 & 160 & 20 & 0 & 1 & & 0.1 \\
\hline 414 & $\mathrm{~B}$ & 71 & 4 & 400 & 30 & 0 & 0 & & 0.3 \\
\hline 415 & $\mathrm{~B}$ & 71 & 4 & 300 & 30 & 0 & 0 & & 0.2 \\
\hline 416 & $71-30$ & 71 & 2 & 3,160 & 40 & 0 & 1 & Ditch work & 2.9 \\
\hline 417 & 7 & 71 & 4 & 2,112 & 30 & 0 & 0 & & 1.5 \\
\hline 418 & Youman Rd & 71 & 2 & 300 & 60 & 0 & 1 & & 0.4 \\
\hline 419 & In 71-24.3 & 71 & 2 & 100 & 60 & 0 & 1 & & 0.1 \\
\hline 420 & $\begin{array}{r}\text { Across } 71- \\
24.3 \mathrm{c} \\
\end{array}$ & 71 & 2 & 2,640 & 40 & 1 & 1 & Ditch work & 2.4 \\
\hline 421 & $71-28$ & 71 & 2 & 2,640 & 40 & 0 & 1 & Ditch work & 2.4 \\
\hline 422 & Turner Rd & 72 & 1 & 60 & 50 & 0 & 1 & & 0.1 \\
\hline 423 & By Turner Rd & 72 & 1 & 0 & 0 & 0 & 1 & 30 acre area & 30.0 \\
\hline 424 & Into $72-23.7$ & 72 & 1 & 850 & 70 & 0 & 1 & & 1.4 \\
\hline 425 & $\mathrm{~B}$ & 43 & 3 & 600 & 15 & 1 & 0 & Steep slopes & 0.2 \\
\hline
\end{tabular}




\begin{tabular}{|c|c|c|c|c|c|c|c|c|c|}
\hline $\begin{array}{c}\text { GIS } \\
\text { Code }\end{array}$ & Road Name & Comp. & Type & $\begin{array}{c}\text { Length } \\
\text { (ft) }\end{array}$ & $\begin{array}{c}\text { Width } \\
\text { (ft) }\end{array}$ & HP & Sed. & Remarks & Acres \\
\hline 426 & 6 & 72 & 1 & 80 & 800 & 1 & 1 & Steep slopes & 1.5 \\
\hline 427 & $72-24$ & 72 & 2 & 160 & 40 & 0 & 1 & Ditch work & 0.1 \\
\hline 428 & $6-1$ & 72 & 2 & 270 & 20 & 0 & 1 & & 0.1 \\
\hline 429 & $6-1$ & 72 & 1 & 1,000 & 70 & 0 & 0 & & 1.6 \\
\hline 430 & $6-1$ & 72 & 1 & 300 & 10 & 0 & 1 & & 0.1 \\
\hline 431 & $72-20$ & 72 & 1 & 3,696 & 60 & 0 & 1 & Ditch work & 5.1 \\
\hline 432 & $6-4.1$ & 70 & 1 & 1,848 & 40 & 0 & 1 & & 1.7 \\
\hline 433 & $6-3$ & 72 & 2 & 450 & 40 & 0 & 1 & & 0.4 \\
\hline 434 & $6-3$ & 72 & 2 & 2,640 & 30 & 1 & 1 & Ditch work & 1.8 \\
\hline 435 & $72-29$ & 72 & 4 & 250 & 40 & 0 & 1 & & 0.2 \\
\hline 436 & $72-29$ & 72 & 1 & 100 & 40 & 0 & 1 & Ditch work & 0.1 \\
\hline 437 & $6-2$ & 72 & 1 & 3,696 & 40 & 1 & 1 & & 3.4 \\
\hline 438 & $6-2$ & 72 & 2 & 100 & 100 & 0 & 1 & Break in dike & 0.2 \\
\hline 439 & $73-20$ & 73 & 1 & 900 & 45 & 0 & 1 & & 0.9 \\
\hline 440 & $\mathrm{~B}$ & 74 & 2 & 2,640 & 20 & 1 & 0 & High walls & 1.2 \\
\hline 441 & $74-32.1 \mathrm{a}$ & 74 & 2 & 200 & 40 & 0 & 0 & & 0.2 \\
\hline 442 & 74-32.1 & 74 & 3 & 100 & 40 & 0 & 1 & & 0.1 \\
\hline 443 & $74-28$ & 74 & 1 & 50 & 10 & 0 & 0 & & 0.0 \\
\hline 444 & $\mathrm{~F}$ & 74 & 3 & 50 & 20 & 0 & 0 & & 0.0 \\
\hline 445 & $\mathrm{~F}$ & 74 & 4 & 100 & 20 & 0 & 0 & & 0.0 \\
\hline 446 & $75-30.2$ & 75 & 2 & 35 & 30 & 0 & 0 & & 0.0 \\
\hline 447 & West of $75-39$ & 75 & 3 & 100 & 20 & 0 & 0 & & 0.0 \\
\hline 448 & $76-21$ & 76 & 1 & 20 & 10 & 0 & 1 & Washout & 0.0 \\
\hline 449 & $76-21$ & 76 & 2 & 200 & 30 & 0 & 1 & Washout & 0.1 \\
\hline 450 & $77-22$ & 77 & 2 & 100 & 40 & 0 & 0 & & 0.1 \\
\hline
\end{tabular}




\begin{tabular}{|c|c|c|c|c|c|c|c|c|c|}
\hline $\begin{array}{c}\text { GIS } \\
\text { Code }\end{array}$ & Road Name & Comp. & Type & $\begin{array}{c}\text { Length } \\
\text { (ft) }\end{array}$ & $\begin{array}{l}\text { Width } \\
\text { (ft) }\end{array}$ & HP & Sed. & Remarks & Acres \\
\hline 451 & $77-22$ & 77 & 1 & 50 & 20 & 0 & 1 & Washed out & 0.0 \\
\hline 452 & $\begin{array}{r}\text { Patterson Mill } \\
\mathrm{Rd}\end{array}$ & 80 & 4 & 15 & 15 & 1 & 0 & & 0.0 \\
\hline 453 & Round Tree Rd & 81 & 3 & 20 & 20 & 0 & 0 & & 0.0 \\
\hline 454 & Round Tree Rd & 81 & 4 & 1,000 & 10 & 0 & 0 & & 0.2 \\
\hline 455 & $82-26$ & 82 & 4 & 1,000 & 20 & 0 & 1 & & 0.5 \\
\hline 456 & 82-23.3a & 82 & 1 & 210 & 30 & 0 & 1 & $\begin{array}{l}\text { Heavy sand; } \\
\text { washout }\end{array}$ & 0.1 \\
\hline 457 & $82-25$ & 82 & 2 & 500 & 30 & 0 & 0 & & 0.3 \\
\hline 458 & $82-25$ & 82 & 2 & 100 & 10 & 0 & 0 & & 0.0 \\
\hline 459 & $82-28.4$ & 82 & 1 & 80 & 10 & 0 & 1 & Washout & 0.0 \\
\hline 460 & 83-25 & 83 & 3 & 50 & 30 & 0 & 0 & & 0.0 \\
\hline 461 & A-18.3 & 83 & 3 & 800 & 30 & 0 & 0 & & 0.6 \\
\hline 462 & Ellis $\mathrm{Rd}$ & 84 & 4 & 300 & 30 & 0 & 1 & & 0.2 \\
\hline 463 & C-85 Boundary & 85 & 2 & 100 & 30 & 0 & 0 & & 0.1 \\
\hline 464 & $85-28$ & 85 & 4 & 200 & 30 & 0 & 1 & & 0.1 \\
\hline 465 & $86-25$ & 85 & 3 & 3,696 & 30 & 0 & 0 & & 2.5 \\
\hline 466 & $85-28.3$ & 85 & 3 & 500 & 30 & 0 & 0 & & 0.3 \\
\hline 467 & $85-20$ & 85 & 3 & 200 & 40 & 0 & 1 & Wetlands & 0.2 \\
\hline 468 & $85-30$ & 85 & 2 & 2,640 & 40 & 0 & 1 & $\begin{array}{l}\text { Washed out } \\
\text { spots }\end{array}$ & 2.4 \\
\hline 469 & $8-25$ & 8 & 4 & 3,168 & 30 & 0 & 1 & $\begin{array}{l}\text { Rd ruts; drainage } \\
\text { problem }\end{array}$ & 2.2 \\
\hline 470 & $\begin{array}{r}\text { ENE of } 8-28 \& \\
8-29\end{array}$ & 8 & 1 & 25 & 60 & 0 & 1 & Deep gully & 0.0 \\
\hline 471 & NW of 8-29 & 8 & 1 & 700 & 40 & 0 & 1 & Ditch work & 0.6 \\
\hline
\end{tabular}

